\title{
Generalization of Kalmar's Proof of Deducibility in Two Valued Propositional Logic into Many Valued Logic
}

\author{
Chubaryan Anahit ${ }^{1}$, Khamisyan Artur ${ }^{2}$ \\ ${ }^{1}$ Department of Informatics and Applied Mathematics, Yerevan State University and Russian-Armenian University, Yerevan, Armenia \\ ${ }^{2}$ Department of Informatics and Applied Mathematics, Yerevan State University, Yerevan, Armenia
}

Email address:

achubaryan@ysu.am (Chubaryan A.), Artur.Khamisyan@gmail.com (Khamisyan A.)

To cite this article:

Chubaryan Anahit, Khamisyan Artur. Generalization of Kalmar's Proof of Deducibility in Two Valued Propositional Logic into Many Valued Logic. Pure and Applied Mathematics Journal. Vol. 6, No. 2, 2017, pp. 71-75. doi: 10.11648/j.pamj.20170602.12

Received: February 13, 2017; Accepted: March 15, 2017; Published: March 22, 2017

\begin{abstract}
This paper focuses on the problem of constructing of some standard Hilbert style proof systems for any version of many valued propositional logic. The generalization of Kalmar's proof of deducibility for two valued tautologies inside classical propositional logic gives us a possibility to suggest some method for defining of two types axiomatic systems for any version of 3-valued logic, completeness of which is easy proved direct, without of loading into two valued logic. This method i) can be base for direct proving of completeness for all well-known axiomatic systems of $k$-valued ( $k \geq 3$ ) logics and may be for fuzzy logic also, ii) can be base for constructing of new Hilbert-style axiomatic systems for all mentioned logics.
\end{abstract}

Keywords: Many-Valued Logics, Hilbert-Style Axiomatic Systems, Completeness of Formal System

\section{Introduction}

Many-valued logic (MVL) as a separate subject was created and developed first by Łukasiewicz [4]. His intention was to use a third, additional truth value for "possible" (or "unknown"). The outcomes of these investigations are, however, the Łukasiewicz's systems, and a series of theoretical results concerning these systems. Essentially parallel to the Lukasiewicz approach, Post [5] introduced the basic idea of additional truth degrees, and applied it to problems of there presentation of functions. Later on, Gödel [6], Jaskowski [7], Kleene [8], Bochvar [9], Belnap [10] and many others continued investigation in this area. In the earlier years of development, this caused some doubts about the use fullness of MVL. In the mean time, however, many interesting applications were found in such fields as logic, mathematics, hardware design, artificial intelligence and some other area sofinformation technologies, therefore the investigations in area of MVL are very actual. Main theoretical results concern to formal systems, which can present different versions of MVL and questions on logical completeness of such systems.

In this paper we focus on the problem of constructing of some standard Hilbert style proof systems for any version of many valued propositional logic. Generalization of Kalmar's proof of deducibility for two valued tautologies inside classical propositional logic [1] gives us a possibility to suggest some method for defining of two types axiomatic systems for any version of 3-valued logic, completeness of which is easy proved direct, without of usually loading into two valued logic [11]. This method i) can be base for direct proving of completeness for all well-known axiomatic systems of $k$-valued $(k \geq 3)$ logics and maybe for fuzzy logic also, ii) can be base for constructing of new Hilbert-style axiomatic systems for all mentioned logics.

First of constructed system based on the logic with one designated value and conjunction, disjunction, implication, defined by Gödel, and negation, defined by permuting the truth values cyclically. Axioms of this system_are generalizations of formulas, using in Kalmar's proof of deducibility for two valued tautologies. Second system is obtained from first one by some restrictions, based on the notions of determinative conjunct and determinative disjunctive normal form (dDNF), introduced by first coauthor for two-valued Boolean functions in [2] and generalized in [3] for 3-valued logic. 


\section{Preminilaries}

\section{1. k-Valued Logic}

Let $E_{k}$ be the $\operatorname{set}\left\{0, \frac{1}{\mathrm{k}-1}, \ldots, \frac{\mathrm{k}-2}{\mathrm{k}-1}, 1\right\}$. We use the well-known notions of propositional formula, which defined as usual from $k$-valued propositional variables $p, q, p_{i}(i \geq 1)$ with values from $E_{k}$, parentheses (,), and logical connectives \&, $\vee, \supset, \neg$, defined as follow:

$\boldsymbol{p} \vee \boldsymbol{q}=\max (p, q), \boldsymbol{p} \& q=\min (p, q), \mathrm{p} \supset \boldsymbol{q}=\left\{\begin{array}{l}1, \text { forp } \leq q \\ q, \text { forp }>q\end{array}\right.$ and negation, defined by permuting the truth values cyclically

$$
\neg \boldsymbol{p}=(((k-1) p+1) \bmod k) /(k-1)
$$

For propositional variable $p$ and $\boldsymbol{\delta}=\frac{i}{\mathrm{k}-1}(0 \leq i \leq k-1)$ we define $p^{\boldsymbol{\delta}}$ as $p$ with $(k-1)-I$ negations.

In considered logic we fix 1 as designated value, so a formula $\varphi$ with variables $p_{l}, p_{2}, \ldots p_{n}$ is called $\boldsymbol{k}$-tautology if for every $\tilde{\delta}=\left(\delta_{1}, \delta_{2}, \ldots, \delta_{n}\right) \in E_{k}^{n}$ assigning $\delta_{j}(1 \leq j \leq n)$ to each $p_{j}$ gives the value 1 of $\varphi$.

Our investigations will be focus on the 3 -valued logic, but can be generalize for $k$-valued $(k \geq 3)$ logics in the future.

\subsection{Determinative Disjunctive Normal Form for 2-Valued Logic}

Here we will use the current concepts of the unit Boolean cube $\left(E^{n}\right)$ for $E=\{0,1\}$, a propositional formula and a classical tautology. The particular choice of a language for presented propositional formulas is immaterial in this consideration. However, because of some technical reasons we assume that the language contains the propositional 2valued variables $p_{i}(i \geq 1)$ and (or) $p_{i_{j}}(i \geq 1 ; j \geq 1)$, logical connectives $\neg, \&, \vee, \supset$ and parentheses (,). Following the usual terminology we call the variables and negated variables literals for 2-valued logic. The conjunct $\mathrm{K}($ term) can be represented simply as a set of literals (no conjunct contains a variable and its negation simultaneously).

In [2] the following notions were introduced.

We call a replacement-rule each of the following trivial identities for a propositional formula $\psi$ :

Here must be deleted repetition of previous sentence

$$
\begin{aligned}
& 0 \& \psi=0, \quad \psi \& 0=0, \quad 1 \& \psi=\psi, \quad \psi \& 1=\psi, \\
& 0 \vee \psi=\psi, \quad \psi \vee 0=\psi, \quad 1 \vee \psi=1, \quad \psi \vee 1=1 \text {, } \\
& 0 \supset \psi=1, \quad \psi \supset 0=\bar{\psi}, \quad 1 \supset \psi=\psi, \quad \psi \supset 1=1 \text {, } \\
& \overline{0}=1, \quad \overline{1}=0, \quad \overline{\bar{\psi}}=\psi \text {. }
\end{aligned}
$$

Application of a replacement-rule to some word consists in replacing some its subwords, having the form of the left-hand side of one of the above identities, by the corresponding right-hand side.

Let $\phi$ be a propositional formula, $P=\left\{p_{1}, p_{2}, \ldots, p_{n}\right\}$ be the set of all variables of $\phi$, and $P^{\prime}=\left\{p_{i_{1}}, p_{i_{2}}, \ldots, p_{i_{m}}\right\}$ ( $1 \leq m \leq n)$ be some subset of $P$.

Definition 2.1. Given $\sigma=\left\{\sigma_{1}, \ldots, \sigma_{m}\right\} \subset E^{m}$, the conjunct $K^{\sigma}=\left\{p_{i_{1}}^{\sigma_{1}}, p_{i_{2}}^{\sigma_{2}}, \ldots, p_{i_{m}}^{\sigma_{m}}\right\}{ }^{1}$ is called $\phi-1$-determinative ( $\phi-0$-determinative) if assigning $\sigma_{j}(1 \leq j \leq m)$ to each $p_{i_{j}}$ and successively using replacement-rules we obtain the value of $\phi(1$ or 0$)$ independently of the values of the remaining variables.

$\phi-1$-determinative conjunct and $\phi-0$-determinative conjunct are called also $\phi$-determinative or determinative for $\phi$.

\subsection{Determinative Disjunctive Normal Form for3-Valued Logic}

Here we recall the notion of determinative disjunctive normal form for 3-valued logic, given in [3] as follow.

Above mentioned replacement-rules are valid for \& and $\mathrm{V}$, but for $\supset$ are valid only $0 \supset \psi=1,1 \supset \psi=\psi$ and $\psi \supset 1=$ 1. For negation the replacement rules are $\neg 0=1 / 2, \neg 1 / 2=1$, $\neg 1=0$ and $\neg \neg \neg \psi=\psi$.

For the other cases we have introduced the following auxiliary relations for replacement

$$
\begin{gathered}
1 / 2 \& \psi=\psi \& 1 / 2 \leq 1 / 2,1 / 2 \vee \psi=\psi \vee 1 / 2 \geq 1 / 2, \\
\psi \supset 0=\neg s g \psi, 1 / 2 \supset \psi=s g \psi, \psi \supset 1 / 2 \geq 1 / 2,
\end{gathered}
$$

Where by $\neg s g \psi$ is denote a function, which is equal to 0 , if value of $\psi$ is more than 0 and 1 , in the opposite case, and by $s g \psi$ is denote the function, which is equal to 1 , if value of $\psi$ is more than 0 , and 0 , in the opposite case.

For every propositional variable $p$ in 3 -valued logic $p, \neg p$ and $\neg \neg p$ are the literals.

Let $\phi$ be a propositional formula of 3-valued logic, $P=\left\{p_{1}, p_{2}, \ldots, p_{n}\right\}$ be the set of all variables of $\phi$, and $P^{\prime}=\left\{p_{i_{1}}, p_{i_{2}}, \ldots, p_{i_{m}}\right\}(1 \leq m \leq n)$ be some subset of $P$.

Definition 3.1. Given $\tilde{\sigma}=\left(\sigma_{1}, \sigma_{2}, \ldots, \sigma_{m}\right) \in E_{3}^{m}$, the conjunct $\quad K^{\sigma}=\left\{p_{i_{1}}^{\sigma_{1}}, p_{i_{2}}^{\sigma_{2}}, \ldots, p_{i_{m}}^{\sigma_{m}}\right\} \quad 2$ is called $\phi-1$ determinative ( $\phi-0$-determinative, $\varphi$-1/2-determinative), if assigning $\sigma_{j}(1 \leq j \leq m)$ to each $p_{i_{j}}$ and successively using replacement-rules and, if it is neccesary, the auxiliary relations for replacement also, we obtain the value of $\phi(1,0$ or $1 / 2$ ) independently of the values of the remaining variables.

For example the conjuncts $p_{l} \neg p_{1}$ и $\neg \neg p_{1}$, are 1determinative for the formula $p_{1} \supset\left(p_{2} \supset\left(p_{3} \supset p_{1}\right)\right)$. For two

\footnotetext{
${ }^{1}$ As usual, given a propositional variable $p$ and $\sigma \in E^{1}$, by $p^{\sigma}$ we denote the function $p^{\sigma}=\left\{\begin{array}{lll}p, & \text { if } & \sigma=1, \\ \bar{p}, & \text { if } & \sigma=0 .\end{array}\right.$

${ }^{2}$ for $p^{\sigma}$ we use here the definition of the point 2.1 .
} 
first we use only replacement-rules, but for the last one we must use the auxiliary relations for replacement also.

Definition of dDNF for3-valued logic gives by analogy. It is easy proved that 1 ) if for some tautology $\phi$ the minimal number of literals, containing in $\phi$-determinative conjunct, is $m$,then $\phi$-determinative DNF has at least $3^{m}$ conjuncts; 2 ) if for some tautology $\phi$ there is such $m$ that every conjunct with $m$ literals is $\phi$-determinative, then there is $\phi$-determinative DNF with no more than $3^{m}$ conjuncts.

By analogy can be define determinative conjuncts and $\mathrm{dDNF}$ for $k$-valued logic with the mentioned properties also. For $k$-valued logic we must introduce the corresponding replacement-rules and auxiliary relations for replacement.

\section{Definitions and Properties of Main Systems}

Here the two main proof systems for above version of 3valued logic are described. The completeness every of them will be proved also.

\subsection{The System CN3 (Cyclically Negation3-Valued)}

For every formula $A, B, C$ of 3 -valued logic and each $\sigma_{1}, \sigma_{2}$ from the set $\{0,1 / 2,1\}$ the following formulas are axioms schemes of $\mathrm{CN} 3$

1.A $\supset(B \supset A)$

2. $(A \supset B) \supset((A \supset(B \supset C)) \supset(A \supset C))$

3. $A^{\sigma_{1}} \supset\left(B^{\sigma_{2}} \supset(A \supset B)^{\varphi_{\supset}\left(A, B, \sigma_{1}, \sigma_{2}\right)}\right)$

4. $A^{\sigma_{1}} \supset\left(B^{\sigma_{2}} \supset(A \vee B)^{\varphi_{\vee}\left(A, B, \sigma_{1}, \sigma_{2}\right)}\right)$

5. $A^{\sigma_{1}} \supset\left(B^{\sigma_{2}} \supset(A \& B)^{\left.\varphi_{\&\left(A, B, \sigma_{1}, \sigma_{2}\right)}\right)}\right.$

6. $A^{\sigma} \supset(\neg A)^{\bar{\sigma}}$

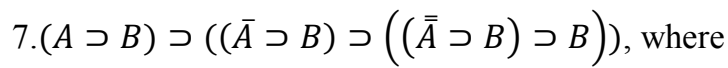

$$
\begin{aligned}
& \varphi_{\supset}\left(A, B, \sigma_{1}, \sigma_{2}\right)=\left(\sigma_{1} \supset \sigma_{2}\right) \&(\neg(A \bigvee \bar{A}) \bigvee(\overline{\bar{B}} \supset \\
& B)) \bigvee(\neg(A \bigvee \overline{\bar{A}}) \& \neg(B \bigvee \overline{\bar{B}})), \\
& \varphi_{\vee}\left(A, B, \sigma_{1}, \sigma_{2}\right)= \\
& \left(\sigma_{1} \bigvee \sigma_{2}\right) \bigvee((A \supset \bar{A}) \& \neg(\bar{B} \bigvee \overline{\bar{B}})) \bigvee(\neg(\bar{A} \bigvee \overline{\bar{A}}) \&(B \supset \bar{B})) \\
& \varphi_{\&}\left(A, B, \sigma_{1}, \sigma_{2}\right) \\
& =\left(\sigma_{1} \& \sigma_{2}\right) \bigvee((A \& \overline{\bar{A}}) \bigvee(B \& \bar{B})) \mathrm{V}((A \& \bar{A}) \bigvee(B \& \overline{\bar{B}})
\end{aligned}
$$

Inference rule is modus ponens /m.p./ $\frac{A, A \supset B}{B}$.

Note that every of schemes 3.-5. Presents at 9 schemes in its turn, and the scheme 6 . presents 3 schemes.

It is important to note that for $A=\sigma_{1}$ and $B=\sigma_{2}$ the following equations $\varphi_{\supset}\left(A, B, \sigma_{1}, \sigma_{2}\right)=\left(\sigma_{1} \supset \sigma_{2}\right)$, $\varphi_{\vee}\left(A, B, \sigma_{1}, \sigma_{2}\right)=\left(\sigma_{1} \bigvee \sigma_{2}\right)$ and $\varphi_{\&}\left(A, B, \sigma_{1}, \sigma_{2}\right)=\left(\sigma_{1} \& \sigma_{2}\right)$ are valid.

It is not difficult to show that all formulas, given by axioms schemes 1.-7. are 3-tautologies. Note, that the functions $\varphi_{*}\left(A_{1}, A_{2}, \sigma_{1}, \sigma_{2}\right)$ play the main role for the axioms schemes 3.-5..

The notions of derivation and derivation from premises are defined as usually.
It is easy to prove the following statement for CN3.

Deduction theorem. Let $\Gamma$ be a set of some formulas and $A$ and $B$ be some formulas. If the formula $B$ is derived in the system $\mathrm{CN} 3$ from the premises $\Gamma$ and $A\left(\Gamma, A \vdash_{C N 3} B\right)$ then the formula $A \supset B$ is derived in the system CN3 from the premises $\Gamma\left(\Gamma \vdash_{C N 3} A \supset B\right)$.

Really, for proving this theorem it must be use the axioms 1. and 2., and also the formula $A \supset A$, which in his turn can be derived using the formulas 1 . and 2 .

Further we will omit the abbreviation $\mathrm{CN} 3$ from the notion $\vdash_{\mathrm{CN} 3}$ sometimes.

Main Lemma Let $P=\left\{p_{1}, p_{2}, \ldots, p_{n}\right\}$ be the set of all variables of any formula $A$, then for every $\tilde{\delta}=\left(\delta_{1}, \delta_{2}, \ldots, \delta_{n}\right) \in E_{3}^{n}$

$$
p_{1}^{\delta_{1}}, p_{2}^{\delta_{2}}, \ldots, p_{n}^{\delta_{n}} \vdash A^{A\left(\delta_{1}, \delta_{2}, \ldots, \delta_{n}\right)} .
$$

This statement is generalization of corresponding Lemma for 2-valued logic (see for example [1]).

Proof is given by induction on number $n$ of variables in the formula $A$. For $n=1$ we have by $\delta=0 \overline{\bar{p}} \vdash \overline{\bar{p}}$, by $\delta=$ $1 / 2 \bar{p} \vdash \bar{p}$ and by $\delta=1 p \vdash p$. Suppose that statement is valid for number of variables $\leq \mathrm{n}$. If the number of variables is $\mathrm{n}+1$, then formula $A$ can be in the one of following forms:

1. $A=A_{1} * A_{2}$,where $* \in\{\&, \mathrm{~V}, \supset\}$,

2. $A=\neg A_{1}$

For the case $1 . A_{1}(\tilde{\delta})=\sigma_{1}, A_{2}(\tilde{\delta})=\sigma_{2}=>A(\tilde{\delta})=\sigma_{1} *$ $\sigma_{2}$

By induction hypothesis

$$
\begin{aligned}
& p_{1}^{\delta_{1}}, p_{2}^{\delta_{2}}, \ldots, p_{n}^{\delta_{n}} \vdash A_{1}^{\sigma_{1}} \\
& p_{1}^{\delta_{1}}, p_{2}^{\delta_{2}}, \ldots, p_{n}^{\delta_{n}} \vdash A_{2}^{\sigma_{2}}
\end{aligned}
$$

Use one of the axiom schemes 3.-5. we have

$p_{1}^{\delta_{1}}, p_{2}^{\delta_{2}}, \ldots, p_{n}^{\delta_{n}} \vdash A_{1}^{\sigma_{1}} \supset\left(A_{2}^{\sigma_{2}} \supset\left(A_{1} * A_{2}\right)^{\varphi_{*}\left(A_{1}, A_{2}, \sigma_{1}, \sigma_{2}\right)}\right)$

And for $A_{1}=\sigma_{1}, A_{2}=\sigma_{2}$

$$
p_{1}^{\delta_{1}}, p_{2}^{\delta_{2}}, \ldots, p_{n}^{\delta_{n}} \vdash A_{1}^{\sigma_{1}} \supset\left(A_{2}^{\sigma_{2}} \supset\left(A_{1} * A_{2}\right)^{\sigma_{1} * \sigma_{2}}\right)
$$

And after two modus ponens derives $\left(A_{1} * A_{2}\right)^{\sigma_{1} * \sigma_{2}}$.

For the case2. $A_{1}(\tilde{\delta})=\sigma=>A(\tilde{\delta})=\bar{\sigma}$ and we must use the axiom scheme 6 .

Corollary. If $\bar{A}$ is 3 -valued tautology, then for every $\tilde{\delta}=\left(\delta_{1}, \delta_{2}, \ldots, \delta_{n}\right) \in E_{3}^{n}$

$$
p_{1}^{\delta_{1}}, p_{2}^{\delta_{2}}, \ldots, p_{n}^{\delta_{n}} \vdash A \text {. }
$$

Theorem. Any formula is derived in CN3 iff it is 3-valued tautology.

Proof. It is obvious that every formula, which is derived in CN3, is 3-valued tautology.

Let $P=\left\{p_{1}, p_{2}, \ldots, p_{n}\right\}(n \geq 1)$ be the set of all variables of any tautology $A$. For every $\tilde{\delta}=\left(\delta_{1}, \delta_{2}, \ldots, \delta_{n}\right) \in E_{3}^{n}$ by above corollary we have $p_{1}^{\delta_{1}}, p_{2}^{\delta_{2}}, \ldots, p_{n}^{\delta_{n}} \vdash A$.

For every $\delta_{1}, \delta_{2}, \ldots, \delta_{n-1}$ we take into consideration the following 3 truth values 


$$
\begin{gathered}
\left\{\begin{array}{c}
\delta_{1}, \delta_{2}, \ldots, \delta_{n-1}, 0 \\
\delta_{1}, \delta_{2}, \ldots, \delta_{n-1}, 1 / 2, \text { for which we have } \\
\delta_{1}, \delta_{2}, \ldots, \delta_{n-1}, 1
\end{array}\right. \\
\left\{\begin{array}{l}
p_{1}^{\delta_{1}}, p_{2}^{\delta_{2}}, \ldots, p_{n-1}^{\delta_{n-1}}, \overline{\bar{p}}_{n} \vdash A \\
p_{1}^{\delta_{1}}, p_{2}^{\delta_{2}}, \ldots, p_{n-1}^{\delta_{n-1}}, \bar{p}_{n} \vdash A . \\
p_{1}^{\delta_{1}}, p_{2}^{\delta_{2}}, \ldots, p_{n-1}^{\delta_{n-1}}, p_{n} \vdash A
\end{array}\right.
\end{gathered}
$$

By deduction theorem we have

$$
\begin{aligned}
& p_{1}^{\delta_{1}}, p_{2}^{\delta_{2}}, \ldots, p_{n-1}^{\delta_{n-1}} \vdash \overline{\bar{p}}_{n} \supset A \\
& p_{1}^{\delta_{1}}, p_{2}^{\delta_{2}}, \ldots, p_{n-1}^{\delta_{n-1}} \vdash \bar{p}_{n} \supset A \\
& p_{1}^{\delta_{1}}, p_{2}^{\delta_{2}}, \ldots, p_{n-1}^{\delta_{n-1}} \vdash p_{n} \supset A .
\end{aligned}
$$

Then adding

$$
\begin{aligned}
& p_{1}^{\delta_{1}}, p_{2}^{\delta_{2}}, \ldots, p_{n-1}^{\delta_{n-1}} \vdash \\
& \left(p_{n} \supset A\right) \supset\left(\left(\bar{p}_{n} \supset A\right) \supset\left(\left(\overline{\bar{p}}_{n} \supset A\right) \supset A\right)\right) / \text { axiom7./ } \\
& p_{1}^{\delta_{1}}, p_{2}^{\delta_{2}}, \ldots, p_{n-1}^{\delta_{n-1}} \vdash\left(\bar{p}_{n} \supset A\right) \supset\left(\left(\overline{\bar{p}}_{n} \supset A\right) \supset A\right) / \text { m.p./ } \\
& p_{1}^{\delta_{1}}, p_{2}^{\delta_{2}}, \ldots, p_{n-1}^{\delta_{n-1}} \vdash\left(\overline{\bar{p}}_{n} \supset A\right) \supset A / \text { m.p./ } \\
& p_{1}^{\delta_{1}}, p_{2}^{\delta_{2}}, \ldots, p_{n-1}^{\delta_{n-1}} \vdash A / \text { m.p./ }
\end{aligned}
$$

So, the number of premises is now $n-1$. Repeating above steps, we obtain finally the derivation of tautology $A$ in CN3. $\square$

Note, that this proof is the full analogy to proof of corresponding theorem for the2-valued logic. It is obvious, that after proving the corresponding Main Lemma for any $k$ valued logic for $k \geq 4$, the proof of the theorem, corresponding above, will be the same. Some hardness for generalization of above results is contained in definition of the functions $\varphi_{*}\left(A, B, \sigma_{1}, \sigma_{2}\right)$ for $* \in\{\&, \mathrm{~V}, \supset\}$ to describing the corresponding axioms schemas 3.-5. for the systems CNk.

\subsection{The System CN3-Cut-Free}

This system is defined by analogy to the cut-free Frege system $F^{-}$, which is described and investigated in [2]. The schematic axioms of the system CN3-cut-free are the following

$$
\text { 1. } \alpha_{1} \&\left(\alpha_{2} \& \ldots \&\left(\alpha_{m-1} \& \alpha_{m}\right) \ldots\right) \supset \alpha_{i}, m \geq 1,1 \leq i \leq m \text {, }
$$$$
\text { 2.a) }\left(K \supset \alpha^{\sigma_{1}}\right) \supset\left(\left(K \supset \beta^{\sigma_{2}}\right) \supset\right.
$$$$
\left.\left(K \supset(\alpha \supset \beta)^{\varphi_{\supset}\left(\alpha, \beta, \sigma_{1}, \sigma_{2}\right)}\right)\right)
$$

$$
\begin{aligned}
& \mathrm{b})\left(K \supset \alpha^{\sigma_{1}}\right) \supset\left(\left(K \supset \beta^{\sigma_{2}}\right) \supset\left(K \supset(\alpha \vee \beta)^{\varphi_{\vee}\left(\alpha, \beta, \sigma_{1}, \sigma_{2}\right)}\right)\right) \\
& \mathrm{c})\left(K \supset \alpha^{\sigma_{1}}\right) \supset\left(\left(K \supset \beta^{\sigma_{2}}\right) \supset\left(K \supset(\alpha \& \beta)^{\varphi_{\&}\left(\alpha, \beta, \sigma_{1}, \sigma_{2}\right)}\right)\right),
\end{aligned}
$$$$
\text { (for functions } \varphi_{\supset}\left(\alpha, \beta, \sigma_{1}, \sigma_{2}\right), \varphi_{\vee}\left(\alpha, \beta, \sigma_{1}, \sigma_{2}\right) \text {, }
$$
$\varphi_{\&}\left(\alpha, \beta, \sigma_{1}, \sigma_{2}\right)$ are defined as above $)$

$$
\text { d) } \left.\left(K \supset \alpha^{\sigma}\right) \supset(K \supset \neg \alpha)^{\bar{\sigma}}\right)
$$

$$
\text { 3.(a) } \quad(\delta \& K \supset \varphi) \supset((\bar{\delta} \& K \supset \varphi) \supset((\overline{\bar{\delta}} \& K \supset \varphi) \supset
$$$$
(K \supset \varphi)))
$$$$
\text { (b) }(\gamma \supset \varphi) \supset((\bar{\gamma} \supset \varphi) \supset((\overline{\bar{\gamma}} \supset \varphi) \supset \varphi)),
$$

where

1. $\varphi$ is provable formula,

2. $\alpha_{i}(1 \leq i \leq m)$ and $\gamma$ are literals, $\alpha, \beta, \delta$ are arbitrary formulas,

3. $K=\beta_{1} \&\left(\beta_{2} \& \ldots \&\left(\beta_{l-1} \& \beta_{l}\right) \ldots\right)(l \geq 1)$ for arbitrary literals $\beta_{i}(1 \leq i \leq l)$,

4. for every $\beta_{1} \&\left(\beta_{2} \& \ldots\left(\beta_{l-1} \& \beta_{l}\right) \ldots\right) \supset \psi$ style subformula from some axiom of second group conjunct $\left\{\beta_{1}, \ldots, \beta_{l}\right\}$ is $\psi$-determinable,

5. if $K^{\text {set }}=\left\{\beta_{1}, \beta_{2}, \ldots, \beta_{n}\right\}$ for some subformula $K=\beta_{1} \& \beta_{2} \& \ldots \& \beta_{k}$ from first axiom of third group, then $\delta \notin K^{\text {set }}$ and $\{\delta\} \cup K^{\text {set }}$ is subset of some $\phi$-determinative conjunct, but $K^{\text {set }}$ is not $\phi$-determinative.

$$
\text { Rule of inference is modus ponens } \frac{A \quad A \supset B}{B} \text {. }
$$

Note that in spite of rule modus ponens, the restrictions1.5."insist on repeats" steps of Main Lemma for derivation of any 3-tautolgy in the system CN3-cut-free. It is obvious that this system is complet.

Note also that on the base of the systems CNk for $k \geq 4$ can be constructed the corresponding systems $\mathrm{CNk}$-cut-free.

\section{Conclusion}

Two types of complete propositional proofs systems for some version of many valued propositional logic are introduced here. In [2] were proved that for 2-tautologies the systems $F^{-}$, resolution and cut-free sequent are polynomially equivalent by proof sizes and by proof steps (polynomial equivalence means, that transformation of any proof in one system into a proof in the other system can be done with no more than polynomial increase of proof complexity). We hope that analogous results can be proved for $k \geq 3$ between the systems $\mathrm{CN} k$-cut-free and $E_{k}$, which are constructed in [3]. It is posible to describe for above version of $k$-valued logic the corresponding cut-free sequent with the same properties. We hope that the systems, correspondings to all systems, which are described here and in [3], can be introduced for other versions of $k$-valued logic.

\section{Acknowledgments}

This work arose in the context of propositional proof complexities research supported by the Russian-Armenian University from founds of MESRF.

\section{References}

[1] E.Mendelson, Introduction to Mathematical Logic, Van Nostrand, Princeton, 1975.

[2] An.Chubaryan, Relative efficiency of some proof systems for classical propositional logic, Proceedings of NASA RA, Vol.37, N5, 2002, and Journal of CMA (AAS),Vol.37, N5, 2002, 71-84.

[3] A.A.Chubaryan, A.S.Tshitoyan, A.A.Khamisyan, On some proof systems for many-valued logics and on proof complexities in it, (in Russian) Reports of NASA RA, Vol. $116, \mathrm{~N} 2,2016,18-24$. 
[4] J.Lukasiewicz, O Logice Trojwartosciowej, Ruch filoseficzny (Lwow), Vol.5, 1920, 169-171.

[5] E.Post, Introduction to a general theory of elementary propositions, Amer. Journ. Math., Vol.43, 1921, 163-185.

[6] K.Godel, Zum intuitionistishen Aussagen kalkul, Akademie der Wissenshaften in Wien, Mathematischenaturwissenschaftliche Klasse, Auzeiger, Vol.69, 1932, 65-66.

[7] S.Jaskowski, On the rules of suppositions in formal logic, Studia logica, No.1, Warsaw, 1934, 5-32.
[8] S.C.Kleene, On the interpretation of intuitionistic number theory, Journ. Symb. Log. Vol.10, No.4, 1945, 109-123.

[9] D.A. Bochvar, M. Bergmann, On a three-valued logical calculus and its application to the analysis of the paradoxes of the classical extended functional calculus, Journal History and Philosophy of Logic, Volume2, Issue1-2, 1981, 87-112.

[10] N.D. Belnap, Auseful four-valued logic, in: Modern Uses of Multiple-valued Logic, Oriel Press., 1977, 35-56.

[11] I.D. Zaslawskij, Symmetrical Konstructive Logic, Press. ASAR, (in Russian), 1978. 